\title{
Parvovirus Diversity and DNA Damage Responses
}

\author{
Susan F. Cotmore ${ }^{1}$ and Peter Tattersall ${ }^{1,2}$ \\ ${ }^{1}$ Department of Laboratory Medicine, Yale University School of Medicine, New Haven, Connecticut 06510 \\ ${ }^{2}$ Department of Genetics, Yale University School of Medicine, New Haven, Connecticut 06510 \\ Correspondence: peter.tattersall@yale.edu
}

Parvoviruses have a linear single-stranded DNA genome, around $5 \mathrm{~kb}$ in length, with short imperfect terminal palindromes that fold back on themselves to form duplex hairpin telomeres. These contain most of the cis-acting information required for viral "rolling hairpin" DNA replication, an evolutionary adaptation of rolling-circle synthesis in which the hairpins create duplex replication origins, prime complementary strand synthesis, and act as hinges to reverse the direction of the unidirectional cellular fork. Genomes are packaged vectorially into small, rugged protein capsids $\sim 260 \AA$ in diameter, which mediate their delivery directly into the cell nucleus, where they await their host cell's entry into $S$ phase under its own cell cycle control. Here we focus on genus-specific variations in genome structure and replication, and review host cell responses that modulate the nuclear environment.

$V$ ruses from the family Parvoviridae are unique in having a linear single-stranded DNA genome, $\sim 5 \mathrm{~kb}$ in length, which terminates in short (120-420 base) imperfect palindromes that fold into self-priming hairpin telomeres. These viruses replicate via a "rolling hairpin" mechanism, with strong evolutionary and mechanistic links to "rolling-circle" replication, as reviewed in detail in previous editions of this work (Cotmore and Tattersall 1996, 2006). Rolling hairpin synthesis relies on the ability of each hairpin to give rise to a duplex origin sequence, which can be nicked by a viral initiator nuclease to create a base-paired DNA primer, and to function as a hinge, allowing quasi-circular synthesis by alternately folding and unfolding to shuttle a continuous unidirectional replication fork back and forth along linear DNA. Together with a few adjacent nucleotides, these palindromes provide all of the cis-acting information required for viral DNA replication and packaging. However, the size, sequence, and predicted structures of the hairpins can vary substantially between genera, or even between the two ends of a single genome, and they appear to have adapted to fulfill multiple additional roles in the life cycle of specific viruses. Parvoviral DNA amplification proceeds via a unidirectional single-strand displacement mechanism through a series of monomeric and concatemeric duplex replicative-form (RF) intermediates, and while the viral initiator protein, variously called NS1 or Rep, serves both as a siteand strand-specific nickase and as a $3^{\prime}$-to- $5^{\prime}$ helicase, all other replicative functions are co-opted from the host cell. This mechanism benefits from

Editors: Stephen D. Bell, Marcel Méchali, and Melvin L. DePamphilis

Additional Perspectives on DNA Replication available at www.cshperspectives.org

Copyright (C) 2013 Cold Spring Harbor Laboratory Press; all rights reserved; doi: 10.1101/cshperspect.a012989

Cite this article as Cold Spring Harb Perspect Biol 2013;5:a012989 
suppression of host DNA synthesis, and generates long stretches of single-stranded DNA with alien terminal structures that invoke host damage responses, which impact both positively and negatively on viral replication. Although details of the mechanisms that mediate parvoviral replication have received relatively little attention since our previous review (Cotmore and Tattersall 2006), recognition that infection is invariably associated with host DNA damage responses (DDRs), some of which are specifically required for efficient viral DNA amplification, has led to significant reappraisal of the nuclear environment and replicative machinery available to these viruses. In parallel, major advances have occurred in our knowledge of genome diversity and cell specificity in this ever-expanding family, which provide novel insight into mechanisms of replication control.

The International Committee on Taxonomy of Viruses (ICTV, Tijssen et al. 2011), classifies viruses in the family Parvoviridae that infect vertebrates as the subfamily Parvovirinae, which currently contains just five genera: the Parvoviruses, Dependoviruses, Amdoviruses, Erythroviruses, and Bocaviruses, although there are at least two additional genera, tentatively called Partetraviruses and Avetalviruses, that await ICTV recognition. This reflects a major jump in known virus diversity, with many new species and genera first identified in clinical or veterinary samples using PCR-based virus discovery methods (Allander et al. 2005; Jones et al. 2005; Day and Zsak 2010). Potential human pathogens that are still pending recognition include genetic variants of Human Bocavirus (HBoV 1-4), which are particularly common in the respiratory and gastrointestinal tracts of children (Kapoor et al. 2010; Kantola et al. 2011), and two broadly distributed genotypes of a "PARV4"-based genus (the aforementioned Partetraviruses), parenterally transmitted among injecting drug users, hemophiliacs, and polytransfused individuals (Sharp et al. 2009; Lahtinen et al. 2011). Most recently, viruses from another potential genus, with sequences resembling both Parvoviruses and Amdoviruses, were detected in fecal samples from children in Burkina Faso, and tentatively named Bufaviruses (Phan et al. 2012). In vitro culture systems or high titer clinical samples are often not available for new members, which can thus only be studied by PCR amplification from patient tissue.

Although the vast majority of parvoviruses replicate without the aid of a helper virus, the adeno-associated viruses (AAVs) from the genus Dependovirus establish latent infections that only become productive when cells are coinfected with a more complex virus, typically an adeno- or herpesvirus. To date the replication mechanisms of adeno-associated virus 2 (AAV2) and minute virus of mice (MVM), from the genus Parvovirus, have been studied in detail, as documented in previous editions of this work. Here we adopt a broader perspective, discussing inter-genera variations that shed light on replication control, and reviewing host cell responses to viral infection that modulate the nuclear environment.

\section{GENOMIC DIVERSITY IN THE PARVOVIRINAE}

Parvoviruses package a single copy of their nonpermuted, single-stranded, linear genome into robust $T=1$ icosahedral protein capsids 18 $26 \mathrm{~nm}$ in diameter, which lack histones or accessory proteins. Viruses from the Dependovirus and Erythrovirus genera are homotelomeric, meaning that their terminal hairpins form part of inverted terminal repeats (ITRs), positioning the same DNA origin sequence at both ends of the genome. These origins are then resolved by equivalent "terminal resolution" mechanisms, ultimately leading to the displacement and encapsidation of DNA strands of both polarities. During terminal resolution the sequence of the hairpin is inverted with each round of synthesis, generating equal numbers of termini with two inverted complementary sequence orientations, termed "flip" and "flop," as detailed previously (Cotmore and Tattersall 1996, 2006). In contrast, members of the Parvo-, Amdo-, and Bocavirus genera are "heterotelomeric," with very different palindromes at the two ends of their genome, which are each processed by different mechanisms and at different rates. In such viruses, the hairpin at the $5^{\prime}$ end of the negative- 
sense strand, by convention called the "right end," is typically resolved by terminal resolution, yielding progeny telomeres in flip and flop orientations. Termini at genomic "left ends," however, are generated from palindromic dimer RF molecules by "asymmetric junction resolution," through a heterocruciform intermediate, a mechanism that conserves a single sequence orientation, denoted flip, at the left end of negative-strand progeny genomes (Cotmore and Tattersall 2003). Although the unique flip sequence could theoretically serve additional critical roles in the viral life cycle, recent studies with viruses carrying axial hairpin mutations indicate that both sequence and orientation changes in the hairpin ears can be tolerated, suggesting that maintaining the flip orientation of these structures is a consequence of, but not the reason for, asymmetric left-end processing. However, the same studies indicated that asymmetric dimer junction resolution is critical for effective MVM replication (Li et al. 2012). In heterotelomeric viruses, imbalance of origin firing typically leads to the preferential displacement of negative-sense progeny single strands from the more potent right-end DNA replication origin, which are then packaged without further selection (Cotmore and Tattersall 2005). Thus, the relative frequency of plus and minus strands and flip and flop termini in packaged DNA provides a useful indicator of the type of resolution mechanism(s) used for their excision.

Genome structures of the type species for recognized genera, and their encoded proteins, are diagrammed in Figure 1. Genera are differentiated primarily by DNA sequence phylogeny, but they are also distinguishable by telomere structure and by the nature and role of the ancillary proteins. Viruses use from one to three transcriptional promoters and one to two polyadenylation signals to coordinate the expression of two major gene complexes, a nonstructural (NS or rep) gene that encodes the viral initiator protein (NS1 or Rep68/78) and a structural (VP or cap) gene. Each genus also encodes a few small ancillary proteins, with disparate functions, variably disposed throughout the genome. In viral origins, the initiator proteins bind site-specifi- cally to two to five variably spaced reiterations of a duplex cognate tetranucleotide motif via oligomeric interactions detailed in Hickman et al. (2004). For viruses in the genus Parvovirus, these clusters of $5^{\prime}$-TGGT-3' repeats are also reiterated in complex patterns at $\sim 100$ base pair intervals throughout the genome, so that, in vivo, NS1 is bound throughout MVM duplex RF DNA (Cotmore et al. 2007). A somewhat similar pattern of extensive $5^{\prime}$-TGGT- $3^{\prime}$ reiteration is also apparent in the newly identified Bufaviruses, suggesting that they share this cognate binding site and chromatin-evasion strategy, but Rep binding site clustering is not seen throughout AAV genomes. In other genera selection experiments to identify preferred oligonucleotide motifs have yet to be reported.

Despite their limited genetic complexity, most parvoviruses replicate productively without the aid of a helper virus, although the absence of viral gene products that induce resting cells to enter $\mathrm{S}$ phase restricts their replication to actively dividing cell populations. To support DNA replication, all telomeres must encode multipartite replication origins and engage in hinge-like hairpin rearrangements, which require local melting induced by site-specific binding of the initiator proteins (Willwand et al. 2002; Lou et al. 2004). In addition, axial asymmetries in the hairpins, most commonly manifest as T- or Y-shaped structures, likely facilitating hairpin unfolding and refolding by lowering the free energy of the duplex. Nevertheless, the complexity and diversity of these hairpins suggests that they fulfill additional roles in the life cycle of specific viruses. For example, in MVM, and potentially in other heterotelomeric viruses, the left-end hairpin contains both symmetric and asymmetric binding sites for transcription factors that modulate expression from the adjacent $\mathrm{P} 4$ promoter (Faisst et al. 1994; Gu et al. 1995; Deleu et al. 1999). More recently, the hairpins of $\mathrm{AAV}$ have been shown to bind MRE11/Rad50/NBS1 (MRN) complexes (Schwartz et al. 2007) and Ku70/ 80 heterodimers (Choi et al. 2010), which are involved in the sensing and repair of DNA damage, raising the possibility that they are tailored to trigger and usurp specific damage responses. 
S.F. Cotmore and P. Tattersall
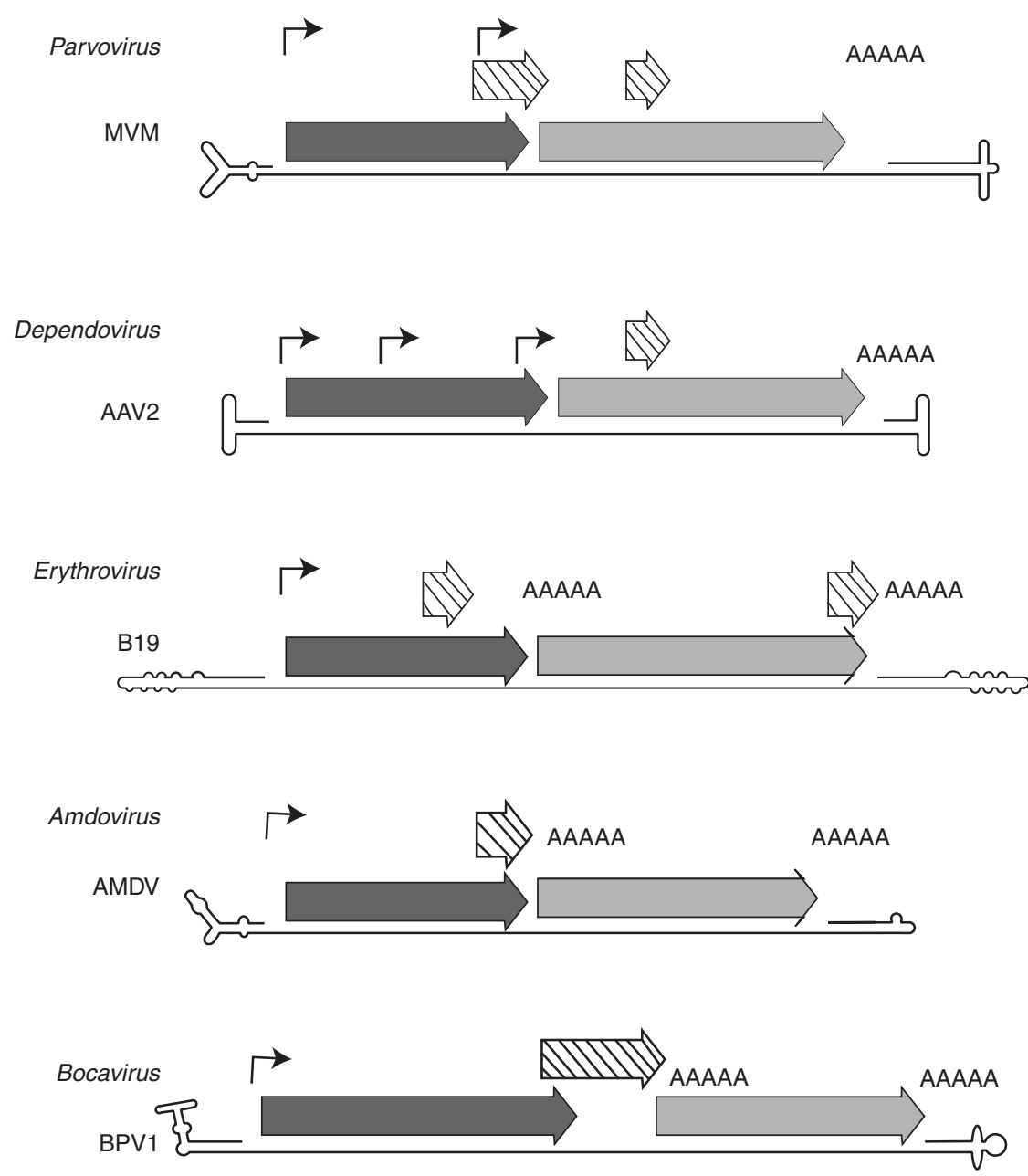

Figure 1. Genetic strategies of representative viruses from genera in the subfamily Parvovirinae. Genomes from the type species of each genus are denoted as a single line terminating in hairpin structures. The hairpins, drawn to represent their predicted structures, are scaled about $20 \times$ with respect to the rest of the genome. Open reading frames are represented by arrowed boxes, shaded dark gray for the major SF3 domain-containing replication initiator protein, light gray for the structural proteins of the capsid, and cross-hatched for sequences unique to the ancillary nonstructural proteins. Ancillary proteins for MVM are NS2 (left) and SAT (right) (Zádori et al. 2005; Ruiz et al. 2011), AAP for AAV2 (Sonntag et al. 2011), $7.5 \mathrm{kDa}$ (left) and $11 \mathrm{kDa}$ (right) for B19 (Zhi et al. 2006), NS2 for AMDV (Oleksiewicz et al. 1996), and NP1 for BPV (Chen et al. 1986). Transcriptional promoters are indicated by solid arrows and polyadenylation sites by the AAAA sequence block.

The Erythrovirus and Bocavirus genera are of particular interest because of their relevance for human disease. As seen in Figure 1, the human erythrovirus B19 encodes three nonstructural proteins: the initiator protein NS1, an early $7.5 \mathrm{kDa}$ species of uncertain function, and an essential $11 \mathrm{kDa}$ late protein (Zhi et al. 2006). It has a homotelomeric genome, with ITRs of 381 nucleotides that terminate in novel, imperfect, 361 nucleotide palindromes. These can potentially fold into long linear duplexes with a few unpaired nucleotides creating a series of small, but highly conserved, mismatched bubbles. B19 shows fastidious tissue specificity, with productive infection restricted to human erythroid progenitor cells (EPCs) between BFU-E (burst- 
forming unit-erythroid) and CFU-E (colonyforming unit-erythroid) stages (Ozawa et al. 1986). Although high titer viremias often occur in vivo, culture systems capable of supporting similar levels of replication remain elusive. Some replication can be induced in nonpermissive 293 cells by cotransfection with adenovirus E2a, E4ORF6, and VA RNA genes (Guan et al. 2009), which are the helper genes typically required for AAV replication. However, these same adenovirus genes enhance replication of many parvovirus-based vectors in $293 \mathrm{~T}$ cells (El-Andaloussi et al. 2011; L Li and P Tattersall, unpubl.), suggesting that they likely elicit common helper mechanisms. More recently, in vivo derived EPCs, expanded in culture to generate $\mathrm{CD}^{+} 6^{+}$cells, have been shown to support substantial B19 replication when reexposed to erythropoietin (Epo). In these cells, stimulation of the Epo/Epo receptor/Janus kinase (Jak2) pathway, or constitutive expression of activated Epo receptors alone, proved sufficient to activate replication of internalized virus (Chen et al. 2010), although progeny virions that could expand infection were still not generated at useful levels. However, culture under hypoxic conditions designed to mimic the normal bone marrow environment enhances both viral DNA replication and progeny production via a $\mathrm{HIF} \alpha-$ independent, STAT5A-mediated pathway (Chen et al. 2011). Exactly how this signaling cascade licenses DNA synthesis remains uncertain, but promises to be of substantial interest.

In contrast to the erythroviruses, the heterotelomeric genomes of bocaviruses in many ways resemble those of the parvoviruses, although NS2 homologs are generally absent and instead bocaviruses encode an abundant $\sim 22.5 \mathrm{kDa}$ nuclear phosphoprotein, called NP1, which is essential for replication. The founding members of this genus, bovine parvovirus (BPV) and minute virus of canines (MVC), can both be propagated efficiently in culture using established cell lines. Following transfection, replication of MVC genomes with a null mutation in NP1 are nonviable, but these can be rescued in trans by NP1 genes from MVC, BPV, or $\mathrm{HBoV} 1$, indicating tightly conserved roles for this protein in different host species (Sun et al. 2009).
Bocaviruses appear to encapsidate predominantly $(90 \%)$ negative-sense DNA with leftend termini that are chiefly $(90 \%)$ in the flip orientation (Chen et al. 1988), suggesting a mixture of resolution strategies in which junction resolution predominates. MVC and BPV genomes have unique hairpins of around 183 and 198 nucleotides at their left and right ends, respectively. When folded to minimize free energy, the MVC left-end hairpin assumes a structure that is somewhat reminiscent of the MVM left end, with a duplex stem in which unpaired nucleotides create a nine residue bubble, a single asymmetric thymidine nucleotide, and axial T-shaped ears, whereas those of BPV contain an additional bubble in place of the asymmetric T. Also akin to MVM, the right-end telomeres of both viruses can be folded into simple duplex structures, which contain internal palindromes that could potentially rearrange to create small cruciform branches near their major dyad axis, as illustrated in Figure 1.

Productive culture systems for human HBoV variants initially appeared elusive, although an important clue for $\mathrm{HBoV}-1$ came from the observation that low levels of viral DNA, detectable by quantitative PCR, were released from pseudostratified human airway epithelium cells following inoculation with HBoV1 PCR-positive nasopharyngeal washes (Dijkman et al. 2009). Remarkably, the entire $\mathrm{HBoV}-1$ genome, complete with hairpins resembling those of MVC and BPV, has now been cloned into bacterial plasmids, and shown to support viral replication and the production of single-round virus stocks following transfection into producer 293 cells (Huang et al. 2012). Such stocks are able to productively infect polarized primary human airway epithelia (HAE) from the apical surface, giving rise to infected HAE that show multiple hallmarks of lung airway-tract injury. Significantly, the 293 cell-derived stocks could also productively infect an immortalized airway epithelial cell line, CuFi-8, but only after these cells had been experimentally induced to polarize. This intriguing and robust reverse genetics system should thus ensure rapid dissection of productive viral replication routes. Given the similarities between the bocaviruses and 
parvoviruses, it will be intriguing to see to what extent their very different NS2 and NP1 ancillary proteins overlap in function.

\section{ESTABLISHING PRODUCTIVE INFECTION}

Parvoviral virions are exceptionally rugged, and while they undergo sequential conformational rearrangements during trafficking through the host cell, most evidence suggests they are ultimately delivered intact directly into the host nucleus (Sonntag et al. 2006). Virions are dense, with single-stranded DNA packed tightly into preformed capsids under control of the viral $3^{\prime}$ to- $5^{\prime}$ helicase (King et al. 2001). Although it has been suggested that nuclear factors mediate disassembly of AAV virions (Thomas et al. 2004), data for MVM suggests that the genome is simply ejected from the otherwise intact capsid, again in a $3^{\prime}$-to- $5^{\prime}$ direction, and then remains tightly associated with it via the genomic $5^{\prime}$ terminus (Cotmore et al. 2010), potentially allowing the capsid to play additional roles in the viral life cycle. For MVM, analysis of capsid mu- tants suggests that both genome packaging and uncoating are mediated by narrow pore-bearing cylindrical structures that surround the icosahedral fivefold axes of the capsid (Plevka et al. 2011; Cotmore and Tattersall 2012), suggesting a highly organized "two portal" model of genome packaging and release, outlined in Figure 2.

Incoming parvovirus virions lack a duplex transcription template or accessory proteins capable of manipulating the cell cycle, and therefore remain quiescent within host cell nuclei until complementary strand DNA synthesis creates the first viral transcription template. For MVM, this duplex is synthesized when the cell enters S phase under its own cell cycle control, and is primed from the left-end hairpin of the negative-sense genome, which is recognized and extended by cellular machinery. The first viral transcripts, driven from the S-phase-responsive P4 promoter in MVM, then allow synthesis of the initiator protein, which ultimately mediates DNA amplification but also acts alongside genus-specific accessory proteins,

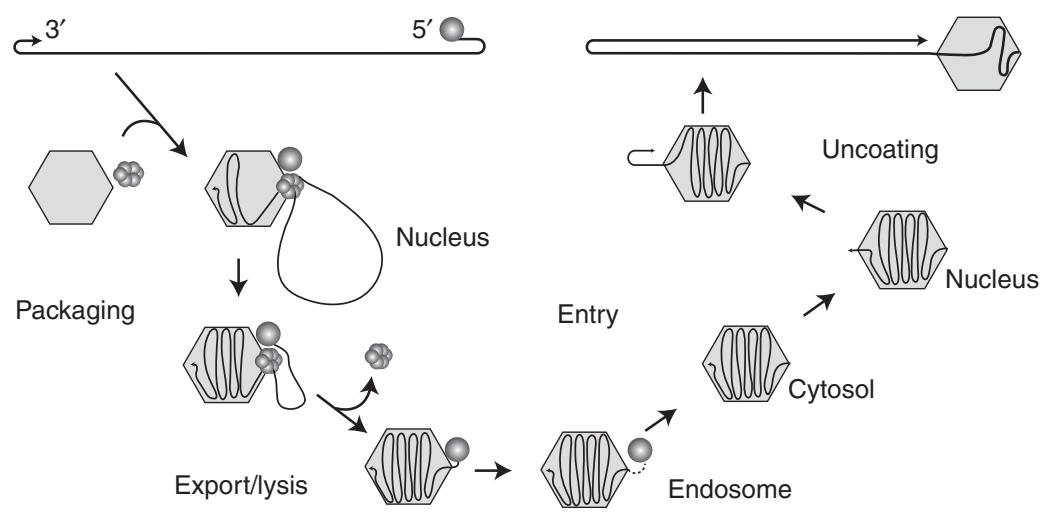

Figure 2. "Two portal" model for genome packaging and uncoating. (Top left) A newly displaced single-stranded progeny genome is depicted $3^{\prime}$-to-5', left-to-right, with a sphere denoting a covalently bound NS1 molecule. Beneath this, the DNA is shown being introduced into a preassembled capsid in a $3^{\prime}$-to- $5^{\prime}$ direction, likely via the helicase activity of an NS1 peptide oligomer (depicted as a hexameric structure, by analogy with AAV) (YoonRobarts et al. 2004), with the $3^{\prime}$ end of the entering genome lodging near the capsid shell, proximal to its destined exit pore. The rest of the strand is then pumped into the particle under increasing pressure, adopting a topology that will allow it to be subsequently unraveled in a $3^{\prime}$-to- $5^{\prime}$ direction. NS 1 is removed from the $5^{\prime}$ end of the DNA during cell entry. $\mathrm{Ca}^{2+} / \mathrm{Mg}^{2+}$ ions in the virion are required to stabilize the pressurized structure, but when these are depleted in vitro, perhaps mimicking a specific host factor trigger in vivo, the $3^{\prime}$ end of the DNA, followed by the coding sequences, is ejected. This uncoated DNA can support complementary strand synthesis, while the $5^{\prime}$ end of the negative strand genome remains threaded with the capsid. 
Parvovirus Diversity and DNA Damage Reponses

such as NS2, to modify the host environment in ways that allow viral replication to proceed.

Where, and in what form, these viruses persist during $G_{0} / G_{1}$ remains enigmatic, because exposed incoming genomes of this type would be expected to invoke cellular DDRs, leading to delays in the $\mathrm{G}_{1}$-S transition and restructuring of the viral DNA. One interesting possibility is that infecting genomes are retained within the virion until specifically induced to uncoat at the start of S phase, thus potentially allowing the capsid to separate the genome from nuclear surveillance mechanisms. Although parvoviruses cannot induce cells to enter $S$ phase, when A9 cells were experimentally synchronized at $G_{1} / S$ using a procedure that allows virus to accumulate in the nucleus before the start of $S$, cellular DNA synthesis rates in MVM-infected cells initially rose, paralleling those in uninfected cells for the first $2 \mathrm{~h}$, but then declined precipitously (Cotmore and Tattersall 1987). Meanwhile, DNA synthesis in uninfected cells peaked between 3 and $5 \mathrm{~h}$ after release, and followed a sharp biphasic curve, beginning to exit $S$ phase after $\sim 8 \mathrm{~h}$. In contrast, in infected cells duplex viral RF first became detectable at $\sim 6 \mathrm{~h}$ after the start of $S$ phase, reached maximal levels by $10 \mathrm{~h}$, and maintained these levels for at least $20 \mathrm{~h}$. This suggests that the presence of virus was detected within $2 \mathrm{~h}$ of entry into $\mathrm{S}$ phase, leading to the cessation of cellular replication. Following a lag period in which the viral nonstructural proteins accumulated, viral DNA amplification then reached peak levels, which were maintained long after uninfected cells entered $\mathrm{G}_{2}$, creating a "pseudo S phase" (Davy and Doorbar 2007) in which viral DNA synthesis continued although host replication had terminated. When DNA content is measured by flow cytometry, this pseudo $S$ phase reads out as sustained supradiploid DNA levels typically scored as "S phase" or " $\mathrm{G}_{2} / \mathrm{M}$ ” cell cycle arrest. Accordingly, $\mathrm{S}$ and $\mathrm{G}_{2} / \mathrm{M}$ phase arrest has been reported following infection with many parvovirus genera, but because a similar flow phenotype can be induced following expression of certain viral nonstructural proteins alone, without ongoing viral DNA replication, interpretation of such data can be complex, as discussed below.

\section{INDUCED DNA DAMAGE RESPONSES}

During infection, replicating viral DNA is typically first observed in a series of discrete foci scattered throughout the nucleus, which are highly enriched for the viral initiator protein NS1/Rep. In synchronized MVM-infected cells NS1 morphology progresses through a series of distinct patterns, as illustrated in Figure 3. Replicating viral DNA first becomes apparent at $\sim 6-8 \mathrm{~h}$ into $\mathrm{S}$ phase, in foci with type II NS1 morphology (Fig. 3C), creating nuclear subdomains called autonomous parvovirus-associated replication (APAR) bodies. These foci recruit cyclin $A$ and various cellular replication proteins known to be involved in viral DNA amplification such as PCNA, RPA, and DNA polymerase $\delta$, but they also sequester DNA polymerase $\alpha$-primase complexes that are not required for MVM replication, so that how specific proteins are recruited to, or excluded from, these sites remains unclear (Cziepluch et al. 2000; Bashir et al. 2001). During this early phase, activated components of various cellular DDR pathways also accumulate within the APAR compartment, as illustrated in Figure $3 \mathrm{G}-\mathrm{I}$, which shows the recruitment of DNAdamage foci containing MDC1 (mediator of DNA-damage checkpoint 1) to the vicinity of APAR bodies in MVM-infected mouse embryonic fibroblasts. Such damage foci also contain $\gamma \mathrm{H} 2 \mathrm{AX}$, which rapidly becomes concentrated within the APAR compartment although MDC1 does not. Similar discrete, but relatively transient, viral replication domains sequestering a spectrum of activated DDR proteins have been observed for many parvoviruses, but exactly which proteins are recruited appears to vary between virus species, and even between cell types. Such foci ultimately expand and coalesce, until accumulating NS1 and viral DNA occupies most of the enlarged nucleus (type IV morphology, Fig. 3E), leaving cellular chromatin compacted and marginated (Ihalainen et al. 2007, 2009).

In general, parvovirus-induced DDRs are characterized by the complex phosphorylation of RPA32, by induction of $\gamma \mathrm{H} 2 \mathrm{AX}$, and by retention of these modified proteins within 


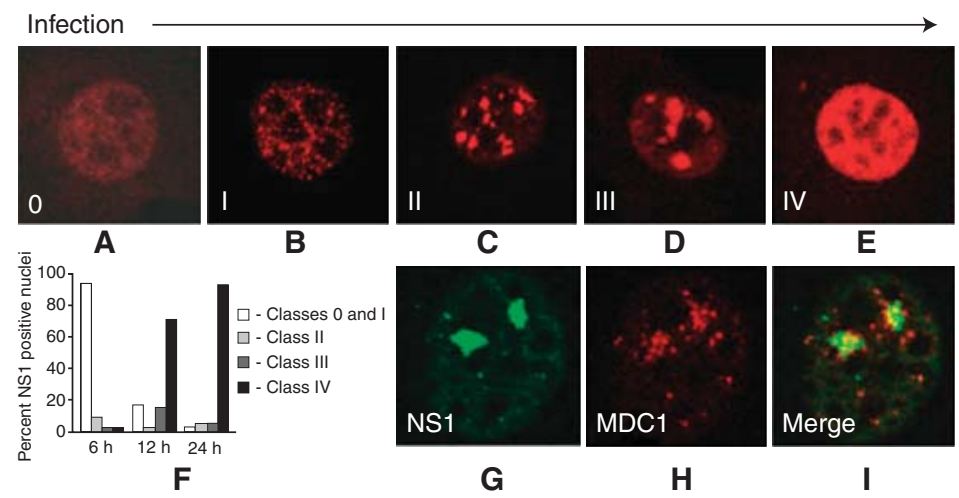

Figure 3. Progressive development of viral replication centers. $(A-F)$ A classification scheme, with classes 0 -IV, shows the progressive changes in NS1 distribution that emerge with time after synchronized A9 cells infected with wild-type MVMp are released into S phase. Classes 0 and I were first observed 3 h into S phase, class II at $6 \mathrm{~h}$, and classes III and IV at $12 \mathrm{~h}$, although the number of cells showing the latter class morphologies progressively increased with time (quantified in F, where the distribution of NS1 morphology classes is plotted against time in seconds). (G-I) Accumulation of MDC1 DDR foci around APAR bodies (stained for NS1) in MVM-infected wild-type mouse embryonic fibroblasts. (From Ruiz et al. 2011; reproduced, with permission, from Elsevier (C) 2011.)

viral replication centers. Each of the three major phosphatidylinositol 3-kinase-like-kinases (PIKKs), ATM, ATR, and DNA-PK, have been implicated in such responses, although details vary. Where tested, pronounced activation of at least one pathway appears required for efficient viral replication, although it is not clear how much this augmentation reflects the direct action of specific DDR components on viral replication mechanisms, and how much simply results from competitive advantages gained by suppression of cellular replication. Thus, for example, infection of murine fibroblasts with MVM potently activates the ATM pathway (Adeyemi et al. 2010; Ruiz et al. 2011), leading to phosphorylation of a number of its downstream substrates and their sequestration within APAR foci. In embryonic mouse fibroblasts, activated ATR and its checkpoint kinase, Chk1, also colocalize with viral replication centers, whereas in A9 fibroblasts, ATR signaling is suppressed in response to infection (Ruiz et al. 2011). Moreover, in human cells, the DNA-PK catalytic subunit and its accessory proteins, Ku70 and Ku86, accumulate in APAR foci. Despite this battery of potential responses, studies with pharmacological inhibitors show that inhibition of the major
ATM response, but not DNA-PK, results in impaired viral DNA replication in MVM-infected A9 cells (Adeyemi et al. 2010).

Similarly, bocavirus MVC infection of canine cells results in activation of both ATM and ATR cascades, but pharmacological inhibition and siRNA knockdown studies show that only ATM activation is required for efficient viral replication and progeny production (Luo et al. 2011a). This could suggest that ATM simply induces a critically intense checkpoint response in these cells, but may also indicate that particular component(s) of the ATM pathway are specifically required. During such infections, MRN complexes were shown to colocalize with replicating viral DNA, and silencing MRE11 inhibited viral replication, which was surprising because MRN complexes have been reported to impair AAV replication (Schwartz et al. 2007). However, MRN fulfills multiple roles in DDR cascades, so that timing of the response may be critical. Accordingly, in MVM infections, MRE11 appeared to colocalize with NS1 at early times, but to be down-regulated via a proteasome-dependent mechanism as infection progressed (Adeyemi et al. 2010), perhaps indicating that MRN plays a positive role in activating 
signaling, but exerts a negative effect during subsequent viral DNA amplification.

By contrast, erythrovirus B19 infection of $\mathrm{CD}^{2} 6^{+}$EPCs resulted in activation of all three major PIKK cascades, leading to recruitment of these kinases and their downstream transducers, Chk2, Chk1, and Ku70/80, to the viral replication compartment. However, in this instance, viral DNA synthesis was impaired by pharmacological inhibitors and shRNAs directed against ATR and DNA-PK, and not by inhibition of ATM (Luo et al. 2011b). Similarly, direct inhibition of Chk1, the ATR-induced checkpoint kinase, sufficed to impair B19 DNA amplification, although whether a dominant negative form of Chk1 could, by itself, promote viral replication during ATR suppression, remains to be explored.

Because viruses rapidly evolve to exploit whatever environments they encounter, it seems likely that specific components of evoked host responses may eventually be co-opted to serve novel roles in viral replication. A possible example of this is seen for AAV. Damage responses invoked by AAVs have been studied in some detail, and found to vary according to the presence or absence of helper virus genes, cell type, and whether the infecting particle contains a viral genome or vector sequence, or has been UV-inactivated (Jurvansuu et al. 2005; Cervelli et al. 2008; Fragkos et al. 2008; Collaco et al. 2009; Schwartz et al. 2009; Choi et al. 2010). However, when replicating productively in the presence of adenovirus helper proteins, they typically induce a DNA-PK-dominated response, suppression of which results in decreased AAV replication. Attempts to identify the cellular factors directly involved in viral replication in vitro, by fractionating crude extracts from adenovirusinfected 293 cells, had suggested that components of the minichromosome maintenance (MCM) complex were absolutely required, perhaps functioning as a replicative helicase (Nash et al. 2008). However, subsequent studies by this group showed that AAV Rep interacts with the Ku70/80 complex (Nash et al. 2009), which serves as the downstream transducer for DNA$\mathrm{PK}$, and that depletion or inactivation of $\mathrm{Ku}$ complexes in crude replication extracts im-
Parvovirus Diversity and DNA Damage Reponses

paired AAV DNA synthesis. These complexes, which can also bind directly to the AAV telomeres, were then shown to support strand-displacement synthesis in vitro, partially substituting for the candidate replicative helicase, MCM. Such observations clearly suggest that current ideas about viral fork structure need to be carefully reexamined in the context of their altered host environment.

\section{CONCLUDING REMARKS}

Previous research efforts, predominantly on MVM and AAV2, focused on viral structures, proteins, and the molecular mechanisms they use to support their unique linear "rolling hairpin" form of unidirectional single-strand displacement synthesis, and on the repertoire of constitutive host factors that underpin this process. More recently, a growing understanding of how cells respond to alien viral DNA structures has shifted this emphasis, refocusing attention on how these inert single-stranded genomes elude recognition while allowing the cell to progress into $S$ phase, and then evoke, evade, and exploit cellular DNA replication and damage responses to promote their own preferential DNA synthesis. Future efforts will likely address how the incoming viral genome establishes transcription and replication complexes, while resisting cellular chromatinization and silencing, and how they usurp control of the $S$ phase replicative and damage-activated pathways to suppress cellular replication while reorganizing the nucleus for optimal virus production. In particular, it should become possible to differentiate between direct effects on the cells metabolic machinery induced by the small number of nonstructural proteins encoded by these viruses, and those resulting from activation of cellular damage pathways evoked by ongoing viral DNA replication.

Modern advances in virus detection methods have revealed the existence of many previously unknown parvoviruses, including entire unsuspected genera and several important human pathogens. Research emphasis will thus likely shift to our expanded knowledge of the viral genomic repertoire that these represent. 
Although some may prove a challenge to propagate in cell culture, the rich diversity of their structures, life cycles, and host cell specificity can be expected to provide opportunities for many new insights into the interactions between this family of simple viral parasites and their host cells.

\section{ACKNOWLEDGMENTS}

This work is supported by Public Health Service grant AI026109 from the National Institutes of Health.

\section{REFERENCES}

Adeyemi RO, Landry S, Davis ME, Weitzman MD, Pintel DJ. 2010. Parvovirus minute virus of mice induces a DNA damage response that facilitates viral replication. PLoS Pathog 6: e1001141.

Allander T, Tammi MT, Eriksson M, Bjerkner A, TiveljungLindell A, Andersson B. 2005. Cloning of a human parvovirus by molecular screening of respiratory tract samples. Proc Natl Acad Sci 102: 12891-12896.

Bashir T, Rommelaere J, Cziepluch C. 2001. In vivo accumulation of cyclin A and cellular replication factors in autonomous parvovirus minute virus of mice-associated replication bodies. J Virol 75: 4394-4398.

Cervelli T, Palacios JA, Zentilin L, Mano M, Schwartz RA, Weitzman MD, Giacca M. 2008. Processing of recombinant AAV genomes occurs in specific nuclear structures that overlap with foci of DNA-damage-response proteins. J Cell Science 121: 349-357.

Chen KC, Shull BC, Moses EA, Lederman M, Stout ER, Bates RC. 1986. Complete nucleotide sequence and genome organization of bovine parvovirus. J Virol 60: 1085-1097.

Chen KC, Shull BC, Lederman M, Stout ER, Bates RC. 1988. Analysis of the termini of the DNA of bovine parvovirus: Demonstration of sequence inversion at the left terminus and its implication for the replication model. J Virol 62: 3807-3813.

Chen AY, Guan W, Lou S, Liu Z, Kleiboeker S, Qiu J. 2010. Role of erythropoietin receptor signaling in parvovirus B19 replication in human erythroid progenitor cells. J Virol 84: 12385-12396.

Chen AY, Kleiboeker S, Qiu J. 2011. Productive parvovirus B19 infection of primary human erythroid progenitor cells at hypoxia is regulated by STAT5A and MEK signaling but not HIF $\alpha$. PLoS Pathog 7: e1002088.

Choi Y-K, Nash K, Byrne BJ, Muzyczka N, Song S. 2010. The effect of DNA-dependent protein kinase on adeno-associated virus replication. PLOS ONE 5: e15073.

Collaco RF, Bevington JM, Bhrigu V, Kalman-Maltese V, Trempe JP. 2009. Adeno-associated virus and adenovirus coinfection induces a cellular DNA damage and repair response via redundant phosphatidylinositol 3-like kinase pathways. Virology 392: 24-33.

Cotmore SF, Tattersall P. 1987. The autonomously replicating parvoviruses of vertebrates. Adv Virus Res 33: 91174.

Cotmore SF, Tattersall P. 1996. Parvovirus DNA replication. In DNA replication in eukaryotic cells (ed. DePamphilis M), pp. 799-813. Cold Spring Harbor Laboratory Press, Cold Spring Harbor, NY.

Cotmore SF, Tattersall P. 2003. Resolution of parvovirus dimer junctions proceeds through a novel heterocruciform intermediate. J Virol 77: 6245-6254.

Cotmore SF, Tattersall P. 2005. Genome packaging sense is controlled by the efficiency of the nick site in the rightend replication origin of parvoviruses minute virus of mice and LuIII. J Virol 79: 2287-2300.

Cotmore SF, Tattersall P. 2006. Parvoviruses. In DNA replication and human disease (ed. DePamphilis M), pp. 593-608. Cold Spring Harbor Laboratory Press, Cold Spring Harbor, NY.

Cotmore SF, Tattersall P. 2012. Mutations at the base of the icosahedral five-fold cylinders of minute virus of mice induce $3^{\prime}$-to- $5^{\prime}$ genome uncoating and critically impair entry functions. J Virol 86: 69-80.

Cotmore SF, Gottlieb RL, Tattersall P. 2007. Replication initiator protein NS1 of the parvovirus minute virus of mice binds to modular divergent sites distributed throughout duplex viral DNA. J Virol 81: 13015-13027.

Cotmore SF, Hafenstein S, Tattersall P. 2010. Depletion of virion-associated divalent cations induces parvovirus minute virus of mice to eject its genome in a $3^{\prime}-$ to- $5^{\prime}$ direction from an otherwise intact viral particle. J Virol 84: 1945-1956.

Cziepluch C, Lampel S, Grewenig A, Grund C, Lichter P, Rommelaere J. 2000. H-1 parvovirus-associated replication bodies: A distinct virus-induced nuclear structure. J Virol 74: 4807-4815.

Davy C, Doorbar J. 2007. G2/M cell cycle arrest in the life cycle of viruses. Virology 368: 219-226.

Day JM, Zsak L. 2010. Determination and analysis of the full-length chicken parvovirus genome. Virology 399: 59-64.

Deleu L, Pujol A, Faisst SR, Rommelaere J. 1999. Activation of promoter $\mathrm{P} 4$ of the autonomous parvovirus minute virus of mice at early $\mathrm{S}$ phase is required for productive infection. J Virol 73: 3877-3885.

Dijkman R, Koekkoek SM, Molenkamp R, Schildgen O, van der Hoek L. 2009. Human bocavirus can be cultured in differentiated human airway epithelial cells. J Virol 83: 7739-7748.

El-Andaloussi N, Endele M, Leuchs B, Bonifati S, Kleinschmidt J, Rommelaere J, Marchini A. 2011. Novel adenovirus-based helper system to support production of recombinant parvovirus. Cancer Gene Ther 18: 240-249.

Faisst SR, Perros M, Deleu L, Spruyt N, Rommelaere J. 1994. Mapping of upstream regulatory elements in the P4 promoter of parvovirus minute virus of mice. Virology 202: 466-470.

Fragkos M, Breuleux M, Clément N, Beard P. 2008. Recombinant adeno-associated viral vectors are deficient in provoking a DNA damage response. J Virol 82: 7379-7387. 
Gu Z, Plaza S, Perros M, Cziepluch C, Rommelaere J, Cornelis JJ. 1995. NF-Y controls transcription of the minute virus of mice $\mathrm{P} 4$ promoter through interaction with an unusual binding site. J Virol 69: 239-246.

Guan W, Wong S, Zhi N, Qiu J. 2009. The genome of human parvovirus b19 can replicate in nonpermissive cells with the help of adenovirus genes and produces infectious virus. J Virol 83: 9541-9553.

Hickman AB, Ronning DR, Perez ZN, Kotin RM, Dyda F. 2004. The nuclease domain of adeno-associated virus rep coordinates replication initiation using two distinct DNA recognition interfaces. Mol Cell 13: 403-414.

Huang Q, Deng X, Yan Z, Cheng F, Luo Y, Shen W, LeiButters DCM, Chen AY, Li Y, Tang L, et al. 2012. Establishment of a reverse genetic system for studying human Bocavirus in human airway epithelia. PLoS Pathog 8: e1002899.

Ihalainen TO, Niskanen EA, Jylhävä J, Turpeinen T, Rinne J, Timonen J, Vihinen-Ranta M. 2007. Dynamics and interactions of parvoviral NS1 protein in the nucleus. Cell Microbiol 9: 1946-1959.

Ihalainen TO, Niskanen EA, Jylhävä J, Paloheimo O, Dross N, Smolander H, Langowski J, Timonen J, Vihinen-Ranta M. 2009. Parvovirus induced alterations in nuclear architecture and dynamics. PLoS ONE 4: e5948.

Jones MS, Kapoor A, Lukashov VV, Simmonds P, Hecht F, Delwart E. 2005. New DNA viruses identified in patients with acute viral infection syndrome. J Virol 79: 82308236.

Jurvansuu J, Raj K, Stasiak A, Beard P. 2005. Viral transport of DNA damage that mimics a stalled replication fork. J Virol 79: 569-580.

Kantola K, Hedman L, Arthur J, Alibeto A, Delwart E, Jartti T, Ruuskanen O, Hedman K, Söderlund-Venermo M. 2011. Seroepidemiology of human bocaviruses 1-4. J Infect Dis 204: 1403-1412.

Kapoor A, Simmonds P, Slikas E, Li L, Bodhidatta L, Sethabutr O, Triki H, Bahri O, Oderinde BS, Baba MM, et al. 2010. Human bocaviruses are highly diverse, dispersed, recombination prone, and prevalent in enteric infections. J Infect Dis 201: 1633-1643.

King JA, Dubielzig R, Grimm D, Kleinschmidt JA. 2001. DNA helicase-mediated packaging of adeno-associated virus type 2 genomes into preformed capsids. EMBO J 20: $3282-3291$.

Lahtinen A, Kivelä P, Hedman L, Kumar A, Kantele A Lappalainen M, Liitsola K, Ristola M, Delwart E, Sharp C, et al. 2011. Serodiagnosis of primary infections with human parvovirus 4, Finland. Emerg Infect Dis 17: 79-82.

Li L, Cotmore SF, Tattersall P. 2012. Maintenance of the flip sequence orientation of the ears in the parvoviral left-end hairpin ears is a non-essential consequence of the critical asymmetry in the hairpin stem. J Virol doi:10.1128/ JVI.01450-12.

Lou HJ, Brister JR, Li JJ, Chen W, Muzyczka N, Tan W. 2004 Adeno-associated virus Rep78/Rep68 promotes localized melting of the rep binding element in the absence of adenosine triphosphate. Chembiochem 5: 324-332.

Luo Y, Chen AY, Qiu J. 2011a. Bocavirus infection induces a DNA damage response that facilitates viral DNA replication and mediates cell death. J Virol 85: 133-145.
Luo Y, Lou S, Deng X, Liu Z, Li Y, Kleiboeker S, Qiu J. 2011b. Parvovirus B19 infection of human primary erythroid progenitor cells triggers ATR-Chk1 signaling, which promotes B19 virus replication. J Virol 85: 8046-8055.

Nash K, Chen W, Muzyczka N. 2008. Complete in vitro reconstitution of adeno-associated virus DNA replication requires the minichromosome maintenance complex proteins. J Virol 82: 1458-1464.

Nash K, Chen W, Salganik M, Muzyczka N. 2009. Identification of cellular proteins that interact with the adenoassociated virus rep protein. J Virol 83: 454-469.

Oleksiewicz MB, Costello F, Huhtanen M, Wolfinbarger JB, Alexandersen S, Bloom ME. 1996. Subcellular localization of Aleutian mink disease parvovirus proteins and DNA during permissive infection of Crandell feline kidney cells. J Virol 70: 3242-3247.

Ozawa K, Kurtzman G, Young N. 1986. Replication of the B19 parvovirus in human bone marrow cell cultures. Science 233: 883-886.

Phan TG, Nguyen PVO, Bonkoungou IJO, Kapoor A, Barro N, O’Ryan M, Kapusinszky B, Wang C, Delwart E. 2012. Acute diarrhea in West-African children: Diverse enteric viruses and a novel parvovirus genus. J Virol doi: 10.1128/JVI.01427-12.

Plevka P, Hafenstein S, Li L, D’Abramo A, Cotmore SF, Rossmann MG, Tattersall P. 2011. Structure of a packaging-defective mutant of minute virus of mice indicates that the genome is packaged via a pore at a 5 -fold axis. $J$ Virol 85: 4822-4827.

Ruiz Z, Mihaylov IS, Cotmore SF, Tattersall P. 2011. Recruitment of DNA replication and damage response proteins to viral replication centers during infection with NS2 mutants of minute virus of mice (MVM). Virology 410: 375-384.

Schwartz RA, Palacios JA, Cassell GD, Adam S, Giacca M, Weitzman MD. 2007. The Mre11/Rad50/Nbs1 complex limits adeno-associated virus transduction and replication. J Virol 81: 12936-12945.

Schwartz RA, Carson CT, Schuberth C, Weitzman MD. 2009. Adeno-associated virus replication induces a DNA damage response coordinated by DNA-dependent protein kinase. J Virol 83: 6269-6278.

Sharp CP, Lail A, Donfield S, Simmons R, Leen C, Klenerman P, Delwart E, Gomperts ED, Simmonds P. 2009. High frequencies of exposure to the novel human parvovirus PARV4 in hemophiliacs and injection drug users, as detected by a serological assay for PARV4 antibodies. J Infect Dis 200: 1119-1125.

Sonntag F, Bleker S, Leuchs B, Fischer R, Kleinschmidt JA. 2006. Adeno-associated virus type 2 capsids with externalized VP1/VP2 trafficking domains are generated prior to passage through the cytoplasm and are maintained until uncoating occurs in the nucleus. J Virol 80: $11040-11054$

Sonntag F, Köther K, Schmidt K, Weghofer M, Raupp C, Nieto K, Kuck A, Gerlach B, Böttcher B, Müller OJ, et al. 2011. The assembly-activating protein promotes capsid assembly of different adeno-associated virus serotypes. J Virol 85: 12686-12697.

Sun Y, Chen AY, Cheng F, Guan W, Johnson FB, Qiu J. 2009. Molecular characterization of infectious clones of the 


\section{S.F. Cotmore and P. Tattersall}

minute virus of canines reveals unique features of bocaviruses. J Virol 83: 3956-3967.

Thomas CE, Storm TA, Huang Z, Kay MA. 2004. Rapid uncoating of vector genomes is the key to efficient liver transduction with pseudotyped adeno-associated virus vectors. J Virol 78: 3110-3122.

Tijssen P, Agbandje-McKenna M, Almendral JM, Bergoin M, Flegel TW, Hedman K, Kleinschmidt JA, Li Y, Pintel DJ, Tattersall P. 2011. Parvoviridae. In Virus taxonomy: Classification and nomenclature of viruses: Ninth report of the international committee on taxonomy of viruses (ed. King AMQ, Adams MJ, Carstens E, Lefkowitz EJ), pp. 375-395. Elsevier, San Diego.

Willwand K, Moroianu A, Horlein R, Stremmel W, Rommelaere J. 2002. Specific interaction of the nonstructural protein NS1 of minute virus of mice (MVM) with
[ACCA $](2)$ motifs in the centre of the right-end MVM DNA palindrome induces hairpin-primed viral DNA replication. J Gen Virol 83: 1659-1664.

Yoon-Robarts M, Blouin AG, Bleker S, Kleinschmidt JA, Aggarwal AK, Escalante CR, Linden RM. 2004. Residues within the $\mathrm{B}^{\prime}$ motif are critical for DNA binding by the superfamily 3 helicase Rep40 of adeno-associated virus type 2. J Biol Chem 279: 50472-50481.

Zádori Z, Szelei J, Tijssen P. 2005. SAT: A late NS protein of porcine parvovirus. J Virol 79: 13129-13138.

Zhi N, Mills IP, Lu J, Wong S, Filippone C, Brown KE. 2006. Molecular and functional analyses of a human parvovirus B19 infectious clone demonstrates essential roles for NS1, VP1, and the 11-kilodalton protein in virus replication and infectivity. J Virol 80: 5941-5950. 


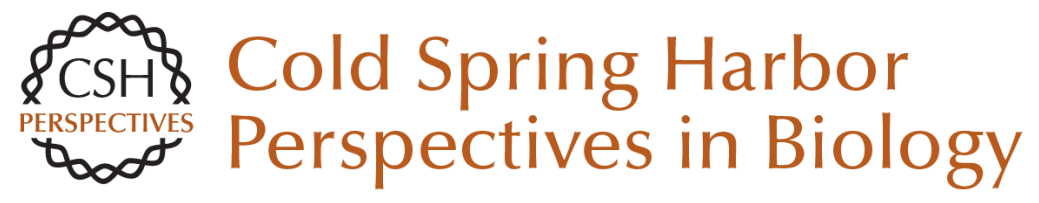

\title{
Parvovirus Diversity and DNA Damage Responses
}

\author{
Susan F. Cotmore and Peter Tattersall
}

Cold Spring Harb Perspect Biol 2013; doi: 10.1101/cshperspect.a012989 originally published online January 4, 2013

\section{Subject Collection DNA Replication}

\section{Replication of Epstein-Barr Viral DNA Wolfgang Hammerschmidt and Bill Sugden}

Replication Proteins and Human Disease Andrew $P$. Jackson, Ronald A. Laskey and Nicholas Coleman

Break-Induced DNA Replication Ranjith P. Anand, Susan T. Lovett and James E. Haber

Regulating DNA Replication in Eukarya Khalid Siddiqui, Kin Fan On and John F.X. Diffley

Archaeology of Eukaryotic DNA Replication Kira S. Makarova and Eugene V. Koonin

Translesion DNA Polymerases Myron F. Goodman and Roger Woodgate

Human Papillomavirus Infections: Warts or Cancer?

Louise T. Chow and Thomas R. Broker

Chromatin and DNA Replication

David M. MacAlpine and Geneviève Almouzni

\author{
Endoreplication \\ Norman Zielke, Bruce A. Edgar and Melvin L. \\ DePamphilis \\ Replication-Fork Dynamics \\ Karl E. Duderstadt, Rodrigo Reyes-Lamothe, \\ Antoine M. van Oijen, et al.
Helicase Activation and Establishment of
Replication Forks at Chromosomal Origins of
Replication
Seiji Tanaka and Hiroyuki Araki
Poxvirus DNA Replication Bernard Moss

The Minichromosome Maintenance Replicative Helicase

Stephen D. Bell and Michael R. Botchan

DNA Replication Origins

Alan C. Leonard and Marcel Méchali

Principles and Concepts of DNA Replication in

Bacteria, Archaea, and Eukarya

Michael O'Donnell, Lance Langston and Bruce Stillman

DNA Replication Timing

Nicholas Rhind and David M. Gilbert

For additional articles in this collection, see http://cshperspectives.cshlp.org/cgi/collection/

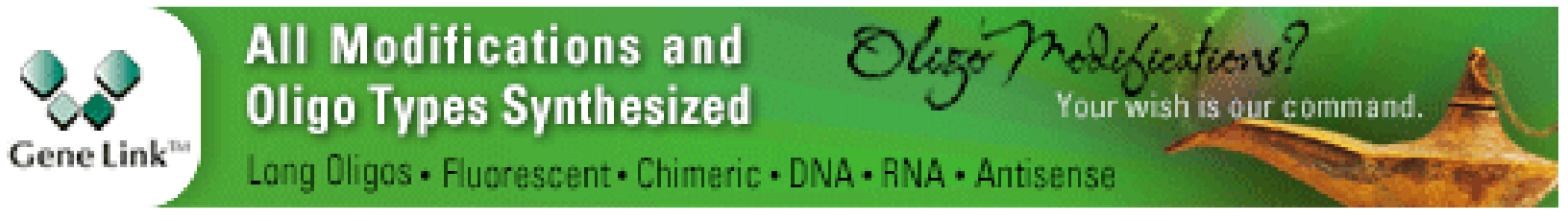

Copyright @ 2013 Cold Spring Harbor Laboratory Press; all rights reserved 